\title{
General and efficient dispersion-based measurement of beam slice parameters
}

\author{
Eduard Prat ${ }^{*}$ and Masamitsu Aiba \\ Paul Scherrer Institut, CH-5232 Villigen PSI, Switzerland
}

(Received 21 January 2014; published 13 March 2014)

\begin{abstract}
In free-electron lasers, it is essential to characterize the beam parameters as a function of the longitudinal position along the bunch, i.e., to determine the so-called slice parameters. Conventional measurements utilize transverse deflector structures to deflect the beam transversely as a function of time. Such structures are expensive and only allow the measurement of slice parameters in one transverse plane. Alternatively, the correlation between time and transverse position can be generated by introducing transverse dispersion to an energy-chirped beam. Dedicated dispersive sections can be used to measure the slice beam parameters in one transverse plane at one location. To extend the applicability of such measurements, we propose to actively generate the dispersion in the two transverse directions wherever required, thus enabling the measurement of beam slice parameters in both transverse planes at potentially any location of the accelerator. Our strategy does not incur any additional cost since it utilizes components already built into the accelerator for other purposes. The application of the method is illustrated with the SwissFEL injector test facility: we show simulations and measurements and we experimentally verify our proposal with the well-established transverse deflector approach.
\end{abstract}

DOI: 10.1103/PhysRevSTAB.17.032801

PACS numbers: 29.20.Ej, 41.60.Cr, 41.85.-p, 29.27.Fh

\section{INTRODUCTION}

In free-electron lasers (FELs), the laser performance such as the radiation power and the temporal profile depends on the electron beam quality-namely the bunch charge, the bunch length, the energy spread, and the transverse emittances. The electron-photon interaction during the lasing process is longitudinally limited to the slippage length (the product of the FEL wavelength and the total number of undulator periods), which typically corresponds to a small fraction of the electron bunch. As an example, at SwissFEL [1], for an electron charge of $200 \mathrm{pC}$ and a radiation wavelength of $1 \AA$, the electron bunch length is about $6 \mu \mathrm{m} \mathrm{rms}$ and the slippage length is about $0.3 \mu \mathrm{m}$. Therefore, to optimize and improve the performance of a FEL facility it is essential to characterize the electron beam not only for the entire bunch but also for each longitudinal fraction of particles on the scale of the slippage length, i.e., to determine the so-called slice parameters.

Emma et al. [2] suggested using a transverse deflector structure (TDS) to measure the slice beam parameters. A TDS deflects the beam in one transverse direction as a function of time so that the slice parameters in the other direction can be reconstructed using two-dimensional profile monitors. In combination with a magnetic spectrometer,

\footnotetext{
*eduard.prat@psi.ch
}

Published by the American Physical Society under the terms of the Creative Commons Attribution 3.0 License. Further distribution of this work must maintain attribution to the author $(s)$ and the published article's title, journal citation, and DOI. the energy distribution along the bunch can also be known. Most existing FEL facilities as well as the planned FEL projects include TDS to measure the slice parameters of the beam with resolutions down to the order of a femtosecond-see for instance Refs. [1,3-6]. Detailed information about slice-parameter measurements using a TDS can be found in Refs. [7,8].

One TDS allows measuring the slice parameters only in the undeflected plane, i.e., either in the horizontal or the vertical direction. In FEL facilities the deflecting direction is normally chosen to be perpendicular to the plane of the bunch compressing chicanes. In this way it is possible to examine the degradation of the beam due to coherent synchrotron radiation (CSR) within the magnetic chicanes.

In the presence of dispersion, a beam is effectively streaked when the longitudinal distribution includes a monotonic correlation between time and energy (an energy chirp). In other words, a two-dimensional beam profile measurement for an energy-chirped beam with finite dispersion is equivalent to a measurement of a beam streaked by a TDS.

Dowell et al. [9] measured the bunch profile and the slice emittance for an energy-chirped beam in a magnetic spectrometer. Slice emittance measurements using dedicated dispersion sections have also been reported for lowenergy electron beams influenced by space-charge [10]. With this setup the measurement can only be done in the nondispersive plane at dispersive locations (magnetic chicanes, collimators, and spectrometers in the case of FELs). For example, the degrading effects due to bunch compression can only be investigated in a dispersive 
section near the bunch compressor with dispersion in the nonbending plane of the magnetic chicane, which is normally not available. To overcome this limitation we generalize the method by actively generating the dispersion in both transverse directions wherever it is necessary, thus enabling the measurement in the two transverse planes at potentially any position of the accelerator.

Early studies measured the slice emittance by using a slit in a dispersive section to select a slice of an energy-chirped beam [11]. A more recent work also uses collimators for slice emittance diagnostics [12]. Apart from being only able to measure at a certain position of the accelerator, these methods have some additional disadvantages due to the fact that the invasive element (or the beam) has to be moved for every slice measurement: the measurement time increases proportionally to the number of beam slices and the energy jitter of the beam may deteriorate the longitudinal resolution. In addition, the wakefields due to the invasive component may compromise the electron beam quality.

Reference [13] suggested the use of skew quadrupoles in a dispersive section to effectively streak the beam at a downstream measurement location, thus replacing the TDS by more affordable skew quadrupoles. The results obtained from this technique were presented in Ref. [14]. The sliceemittance measurement mentioned in Ref. [13] gives, however, a wrong result, since skew quadrupoles introduce a cross-plane coupling (horizontal-vertical) that causes an increase of the observed emittance. We examine with simulation and measurements different ways to obtain the dispersion necessary for the measurement without degrading the beam quality or affecting the measurement results. In particular, we find that orbit bumps can be used to generate dispersion in the two transverse planes at multiple locations along the accelerator.

In contrast to the TDS method, the slice length in the dispersion-based method is determined only relative to the entire bunch length, while the absolute bunch length remains unknown. The absolute bunch length may, however, be measured with longitudinal diagnostics or by the rf-phasing method [15-17], where the longitudinal phase space is retrieved by measuring the energy spectrum as a function of the linac phase. One may use the dispersionbased measurement as a complement to the TDS measurement, that is, measuring the longitudinal beam profile and the slice emittance in one plane with the TDS and the slice emittance in the other plane with the dispersion-based measurement. In this way, we can fully characterize the electron bunch in all six dimensions.

In Sec. II we present the dispersion-based method to measure the slice emittance. In Sec. III we show an application of the method at the SwissFEL injector test facility [18]. Both simulations and measurements, including a comparison with a conventional TDS measurement, are presented.

\section{DISPERSION-BASED SLICE-EMITTANCE MEASUREMENTS}

A slice-emittance measurement is realized by streaking the beam and measuring for different optics the slice beam sizes in the unstreaked direction.

\section{A. Beam streaking}

Conventionally a TDS is employed for the streaking. For a beam with a monotonic correlation between longitudinal position and energy, a finite dispersion function at the measurement location expands the beam size in one dimension, which is equivalent to a beam streaking (see Fig. 1). The streaking will be linear only if the energy chirp is also linear.

In FELs, an energy chirp with typically about $1 \%$ rms energy spread is introduced for enabling bunch compression with a magnetic chicane to achieve the peak current required for the lasing process. Since the energy chirp is not immediately removed after the bunch compressor, the measurement can be done downstream of the magnetic chicane. A quadratic component of the energy chirp is usually compensated with higher-harmonic rf, and the energy chirp after the bunch compressor is then appropriate for the measurement. The energy chirp may also be introduced specifically for the measurement purpose at other locations. If a phase far off-crest or even close to the zero phase is set to one or more accelerating rf structures, an almost linear energy chirp may be obtained without higher harmonic rf.
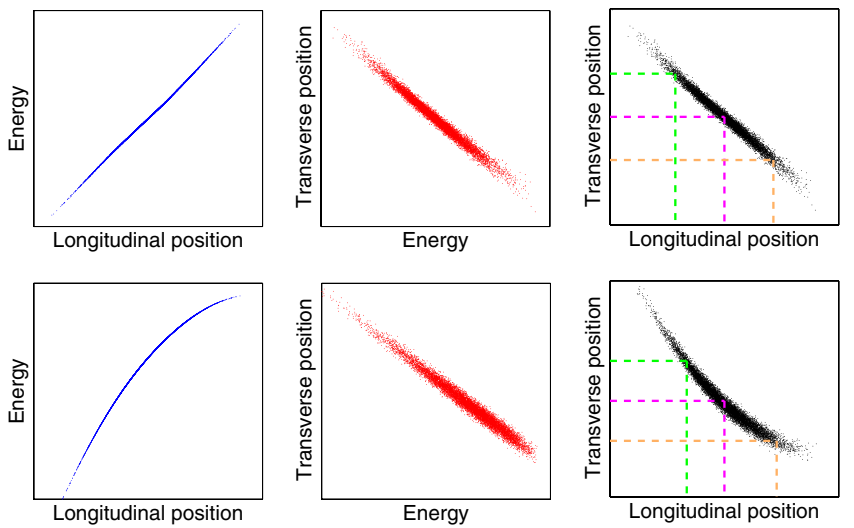

FIG. 1. Beam streaking with dispersion function for an energychirped beam (schematic). The combination of an energy chirp (left plots) and transverse dispersion (center plots) results in an effective beam streaking (right plots). The upper plots show the case where the chirp is linear, the lower plots correspond to a nonlinear energy chirp. The dashed lines in the left plots indicate the relation between transverse and longitudinal position for three parts of the bunch which are equally separated in transverse position. Only in the case of a linear energy chirp will the three regions be equally separated in longitudinal position as well, i.e., only a linear chirp results in a linear streaking. 
There are several ways to introduce the dispersion necessary for the measurement. The dispersion is zero at most locations by design. Exceptions are dispersive sections such as bunch compressors and energy spectrometers, where the slice-emittance measurement is enabled for the nondispersive plane. If a quadrupole is situated in a dispersive section, an angular dispersion may be generated at its location. Therefore, a quadrupole magnet in a bunch compressor allows us to generate the necessary dispersion at a downstream measurement location.

More generally, an orbit bump introduced with dipole corrector magnets over quadrupoles may be used to generate finite dispersion. This approach works for both planes. Since dipole correctors and quadrupoles fulfill essential functions (trajectory/dispersion correction and beam focusing) they are available almost everywhere along the accelerator. Therefore, our strategy is very general and can be applied to both transverse planes at several locations along the accelerator.

\section{B. Longitudinal resolution}

We derive an expression for the longitudinal resolution, i.e., how many slices can be meaningfully measured for a given bunch length. In the following we assume that the dispersion is generated in the horizontal $(x)$ plane and that vertical $(y)$ slice emittances are reconstructed. The same expressions are valid for finding horizontal slice emittances simply by exchanging the planes.

The streaked (horizontal) beam size at the measurement location is determined by the betatronic beam size and the dispersive beam size due to the correlated energy spread (see Fig. 1). We assume that the dispersive beam size due to the uncorrelated (or slice) energy spread is negligible, which is normally the case for FEL electron beams.

Assuming further that the profile monitor resolution is negligibly small compared to the streaked beam size, the longitudinal resolution with respect to the $\mathrm{rms}$ bunch length, $R$, is determined by the ratio of the betatronic beam size $\sigma_{x}$ and the dispersive beam size $\sigma_{D}$ :

$$
R=\frac{\sigma_{x}}{\sigma_{D}}
$$

A ratio of $R=0.1$, for example, means that 10 slices per bunch length are measurable.

The betatronic beam size is given by

$$
\sigma_{x}=\sqrt{\beta_{x} \epsilon_{x}}
$$

where $\beta_{x}$ is the horizontal $\beta$-function at the measurement location, and $\epsilon_{x}$ is the horizontal $\mathrm{rms}$ emittance. The dispersive beam size can be obtained as

$$
\sigma_{D} \approx D_{x} \delta
$$

where $D_{x}$ is the horizontal dispersion function and $\delta$ is the relative momentum deviation (approximated by the relative energy spread assuming a relativistic beam).

The resolution may not be constant along the bunch due to variations in the betatronic beam size from slice to slice. It can also change if there is a nonlinear correlation between longitudinal coordinate and energy (see Fig. 1).

If the measurement location is in a dispersive section such as in a bunch compressor or in a spectrometer, the horizontal $\beta$-function should be minimized to optimize the resolution. In cases where the dispersion is generated by quadrupoles in a dispersive section or by orbit bumps, the dispersion at the measurement location is

$$
D_{x}=\sum_{i} D_{x, i}^{\prime} \sqrt{\beta_{x, i} \beta_{x}} \sin \mu_{x, i},
$$

where $D_{x, i}^{\prime}$ is the angular dispersion generated at the $i$ th quadrupole, $\beta_{x, i}$ is the horizontal $\beta$-function at the $i$ th quadrupole, and $\mu_{x, i}$ is the horizontal phase advance from the $i$ th quadrupole to the measurement location. The resolution is then

$$
R=\frac{\sqrt{\epsilon_{x}}}{\delta \sum_{i} D_{x, i}^{\prime} \sqrt{\beta_{x, i}} \sin \mu_{x, i}} .
$$

The $\beta$-function at the measurement location is not relevant for the resolution. The goal is to design the optics to maximize the sum in the denominator. When the dispersion is generated with a single source (a single quadrupole), the $\beta$-function at the quadrupole location should be maximized and the phase advance between the quadrupole and the measurement position should be close to $\pi / 2+n \pi$ (with $n$ being an integer number).

\section{Determination of the emittance}

The vertical rms emittance in general is defined with the second-order moments of the beam:

$$
\epsilon_{y}=\sqrt{\left\langle y^{2}\right\rangle\left\langle y^{\prime 2}\right\rangle-2\left\langle y y^{\prime}\right\rangle},
$$

where $y$ is the vertical particle position and $y^{\prime}=d y / d s$ is the vertical angle. The so-called normalized emittance is often used as an invariant and is defined as

$$
\epsilon_{n, y}=\frac{p_{0}}{m c} \epsilon_{y}
$$

where $p_{0}$ is the central momentum of the beam, $m$ is the particle mass, and $c$ is the speed of light.

Using a matrix formalism $[19,20]$, the beam size at a position $s$ can be expressed as a function of the secondorder moments of the beam at an upstream location $s_{0}$ and the transfer matrix elements between $s_{0}$ and $s$ : 


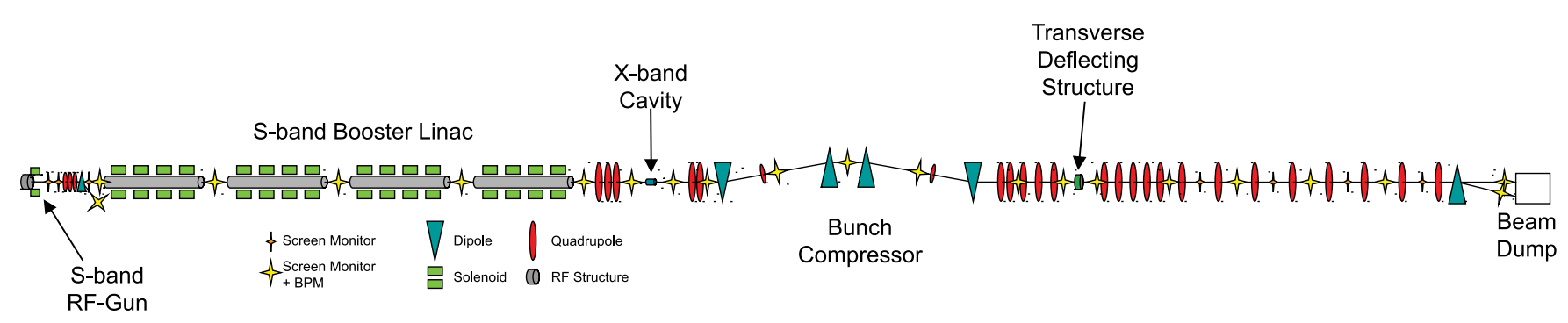

FIG. 2. Schematic layout of the SITF (not to scale). The total length is about $60 \mathrm{~m}$.

$$
\left\langle y^{2}\right\rangle_{s}=R_{33}^{2}\left\langle y^{2}\right\rangle_{s_{0}}+R_{34}^{2}\left\langle y^{\prime 2}\right\rangle_{s_{0}}+2 R_{33} R_{34}\left\langle y y^{\prime}\right\rangle_{s_{0}} .
$$

The second-order moments of the beam can be reconstructed at $s_{0}$ by measuring the beam size at $s$ for varying optics between these locations. A minimum of three measurements (i.e., three equations) are needed to reconstruct the three second-order moments of the beam. More measurements will allow for a more robust reconstruction. The emittance and the Twiss parameters are found from the second-order moment values that best fit the measured beam sizes in a least-squares procedure. To reconstruct the slice emittances (and the slice Twiss parameters) the slice beam sizes are measured for the streaked beam using a twodimensional profile monitor.

We reconstruct the slice emittance using a multioptics approach (similar to Ref. [7]): the beam sizes are measured at a single location and multiple quadrupoles are used to generate the required optics for the measurement. Ideally, the betatron phase advance $\mu_{y}$ between the measurement and reconstruction points should be scanned progressively between 0 and $\pi$ for the best reconstruction of the emittance. Additionally, the longitudinal resolution should be optimized and the $\beta$-function in both planes should be kept within certain limits to avoid issues related to the dynamic range and the resolution of the profile monitor. The optics control is discussed in more detail in Sec. III.

We note that for low-energy beams affected by space charge the optics linearity assumed in Eq. (8) might not be valid. In this case, slit or pepper-pot measurements are more appropriate than optics-based methods. Examples of such measurements can be found for instance in Refs. [10] and [21].

\section{APPLICATION AT THE SWISSFEL INJECTOR TEST FACILITY}

The SwissFEL project [1] planned at the Paul Scherrer Institute foresees the realization of an X-ray FEL with a radiation wavelength down to $0.1 \mathrm{~nm}$. The SwissFEL Injector Test Facility (SITF) [18] is a $250 \mathrm{MeV}$ facility with the main goal of demonstrating the high brightness electron beam required to drive the SwissFEL accelerator. The SITF further serves as a platform for the development and validation of accelerator components needed for the SwissFEL facility.
Electron bunches are generated in a laser-driven rf gun with a nominal bunch charge varying between $10 \mathrm{pC}$ and 200 pC. After the gun section, the beam can be accelerated to its nominal energy of $250 \mathrm{MeV}$ with four S-band accelerating structures working at a frequency $f \approx 3 \mathrm{GHz}$. A bunch compressor chicane after the S-band structures can compress the electron beam longitudinally. An X-band system working at the fourth harmonic of the S-band structures $(f \approx 12 \mathrm{GHz})$ is presently used to linearize the compression but was not available during the measurements presented in the following. An S-band TDS with a maximum integrated voltage of $5 \mathrm{MV}$ is installed after the bunch compressor for diagnostic purposes. The TDS streaks in the vertical direction, so the slice emittance can be reconstructed only in the horizontal plane. After the TDS there is a dipole spectrometer that allows measuring the momentum properties of the beam. Figure 2 shows a sketch of the SITF, where the most relevant elements are indicated.

For all the simulations and measurements presented in this paper, the energy chirp is created by going far off-crest in the third and fourth S-band structures ${ }^{1}$-this assures an almost linear energy chirp.

\section{A. Dispersion generation}

The vertical dispersion is created with an orbit bump using four dipole correctors and five quadrupoles in the region between the bunch compressor and the TDS. We have designed an orbit bump such that at the exit the trajectory is closed and the vertical dispersion functions have the values $D_{y}=-6.8 \mathrm{~mm}, D_{y^{\prime}}=-4.1 \mathrm{mrad}$. The bump amplitude is limited by the available strength of the corrector magnets corresponding to $2 \mathrm{mrad}$ at an energy of $250 \mathrm{MeV}$. Figure 3 shows the calculated trajectory and dispersion functions along the orbit bump.

We use a quadrupole magnet in the bunch compressor to generate the dispersion in the horizontal plane. The bunch compressor is equipped with two quadrupoles to compensate the $x$ - $t$ correlation which can be generated due to CSR effects in the bunch compressor [22]. We use the first

\footnotetext{
${ }^{1}$ In the simulations and the horizontal emittance measurement the chirp was generated by locating the beam at the zero-crossing of the structures, while for the vertical emittance measurement we set the rf phase to 40 degrees off from on-crest acceleration.
} 


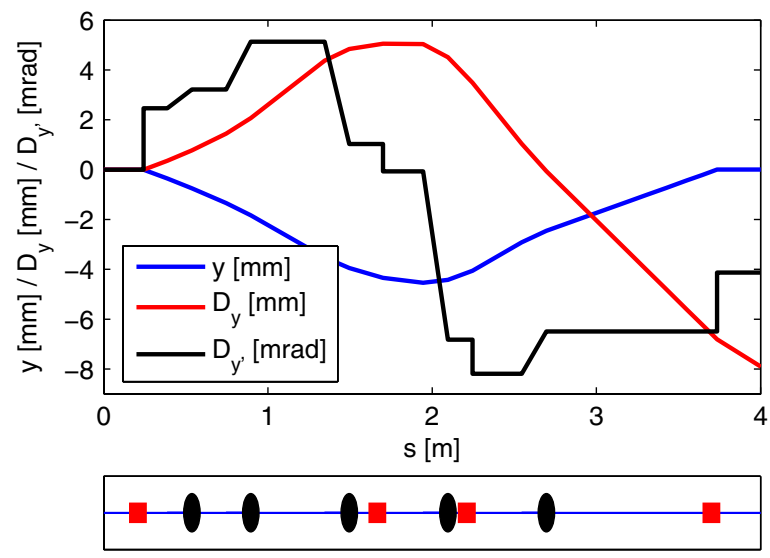

FIG. 3. Vertical dispersion generation with an orbit bump. The upper plot shows the calculated vertical trajectory and dispersion functions through the orbit bump. The lower plot shows a sketch of the lattice: the black ellipses indicate the quadrupole magnets and the red rectangles correspond to the vertical corrector magnets.

quadrupole to generate the dispersion because the $\beta$ function at its position $(\approx 15 \mathrm{~m})$ is larger than at the second quadrupole $(\approx 4 \mathrm{~m})$, which, according to Eq. (5), results in a better longitudinal resolution. The dispersion kick generated by the quadrupole can be calculated as $D_{x^{\prime}}=D_{x} k l$, where $D_{x}$ is the dispersion value at the quadrupole position and $k l$ is the integrated normalized gradient along the quadrupole. The nominal dispersion at the position of the quadrupole, achieved when the bunch compressor dipoles are set to their nominal deflecting angle of 4.1 degrees, is about $100 \mathrm{~mm}$. Figure 4 shows the calculated absolute horizontal trajectory and dispersion functions along the bunch compressor for an integrated normalized gradient of

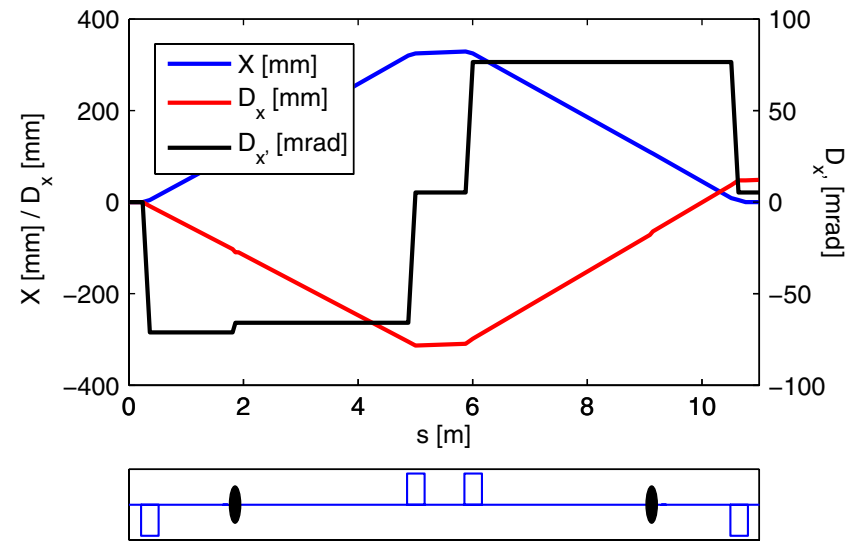

FIG. 4. Horizontal dispersion generation. Calculated absolute horizontal trajectory and dispersion functions through the bunch compressor when the first quadrupole in the bunch compressor has an integrated normalized gradient of $0.05 \mathrm{~m}^{-1}$. The lower plot shows a sketch of the bunch compressor: the blue rectangles correspond to the dipole magnets and the black ellipses correspond to the quadrupoles.
$0.05 \mathrm{~m}^{-1}$, for which the dispersion kick will be approximately 5 mrad.

\section{B. Optics for the slice-emittance measurements}

The required optics for the measurement are generated with five quadrupole magnets with a length of $0.15 \mathrm{~m}$ each. We simultaneously need to scan the phase advance in the measurement direction, to optimize the resolution in the dispersive plane, and to control the $\beta$-functions at the measurement location. We have calculated the required quadrupole strengths to perform a dispersion-based slice measurement by using the code ELEGANT [23]. We limit the strength of the quadrupoles to $\pm 4 \mathrm{~m}^{-2}$ to avoid significant chromatic effects. The optics are controlled in the following way:

(i) Phase advance in the measurement direction: The phase advance in the nondispersive plane is regularly scanned in steps of 5 degrees to cover a total of about 150 degrees.

(ii) Longitudinal resolution (see Eq. (5)): For the calculation of the resolution we assume a correlated energy spread of $1 \%(\delta=0.01)$, a beam energy of $200 \mathrm{MeV}$, and a normalized emittance of $500 \mathrm{~nm}$. For the vertical measurement, the phase advance in the dispersive plane $\mu_{x}$ between the dispersive source and the profile monitor is controlled such that $\sin \mu_{x}>0.95$ for the whole scan. Considering that $\beta_{x, 1} \approx 15 \mathrm{~m}$ this is enough to resolve about five

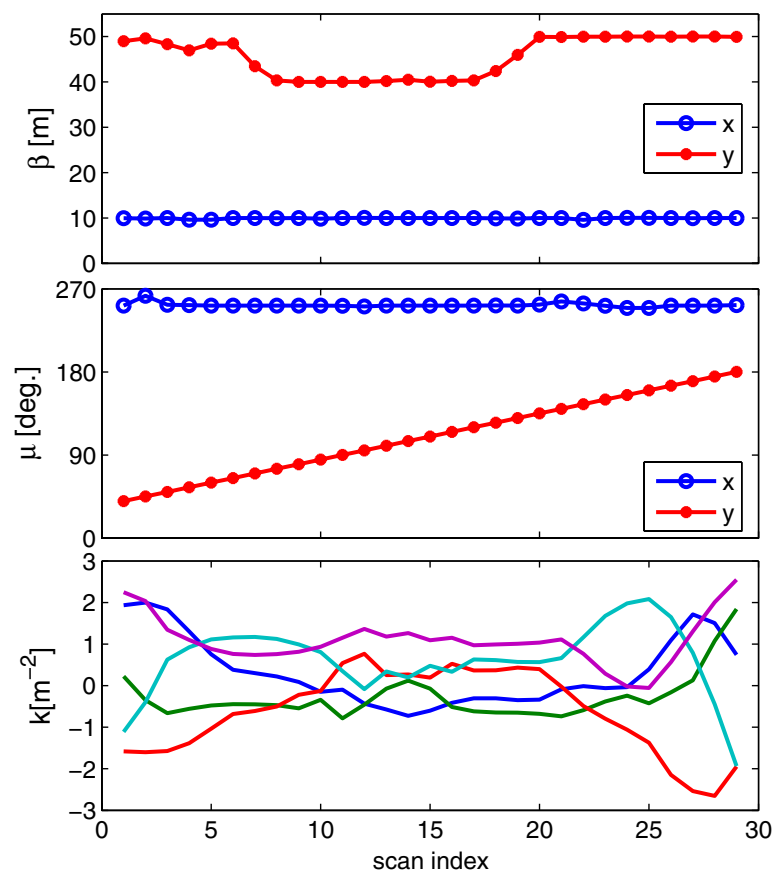

FIG. 5. Scan parameters for the vertical slice-emittance measurement: $\beta$-functions at the measurement position (upper plot), phase advances (middle plot), and quadrupole strengths (lower plot) as a function of the scan index. 
slices per bunch length $(R<1 / 5)$ if the dispersive kick is $5 \mathrm{mrad}$ (the maximum kick is about $25 \mathrm{mrad}$, so the minimum achievable resolution is about $R \approx 0.04$ ). For the horizontal measurement, the maximum orbit bump and the downstream optics allow us to resolve more than six slices per bunch length $(R<1 / 6)$.

(iii) Beam size at the measurement location: The $\beta$ function in the measurement direction is kept between 10 and $50 \mathrm{~m}$ for the whole scan to have beam sizes that can be measured without problems. The $\beta$-function in the dispersive plane is kept below $10 \mathrm{~m}$ to keep under control the beam size in the streaked direction.

As an example, Fig. 5 shows the scan parameters for the vertical slice-emittance measurements. It should be noted that the value of the phase advances and $\beta$-functions will be exactly valid only for the core of the bunch with the nominal energy and will slightly differ for the parts of the bunch with different energies.

\section{Emittance resolution and errors}

The profile monitor used for the measurements is a yttrium-aluminum-garnet (YAG) screen with a measured resolution of about $15 \mu \mathrm{m}$. Our simulations show that this corresponds to a normalized emittance resolution of about 1-2 $\mathrm{nm}$ for an energy of $250 \mathrm{MeV}$.

We performed simulations for $5 \times 10^{4}$ different seeds to evaluate the statistical errors of the reconstructed parameters. Assuming a 5\% error (rms) on the beam-size measurement, the statistical error of the reconstructed emittance is $2.3 \%$ for the horizontal and $1.7 \%$ for the vertical measurement, for the calculated optics.

The calibration error of the profile monitor is of the order of $1 \%$ [24], which causes a systematic error of the reconstructed emittances of around $2 \% .^{2}$ The calibration uncertainty is dominant over other systematic errors such as energy uncertainties or quadrupole field errorsthe energy error is around $0.1 \%$ according to the accuracy of our beam momentum measurements, and the quadrupole field error is about $0.2 \%$ according to the magnetic field measurements done in the lab. We take into account the individual energy of each slice to prevent an additional growth of the systematic errors.

\section{Virtual measurements}

We have simulated the dispersion-based slice-emittance measurement at the SITF with the code ELEGANT [23]. The simulated lattice starts at the entrance of the third S-band rf structure and ends at the location of the profile monitor.

\footnotetext{
${ }^{2}$ Considering that the beam size is proportional to the square root of the emittance $(\sigma=\sqrt{\beta \epsilon})$, by error propagation it can be derived that the error on the emittance is approximately two times the beam-size measurement error.
}
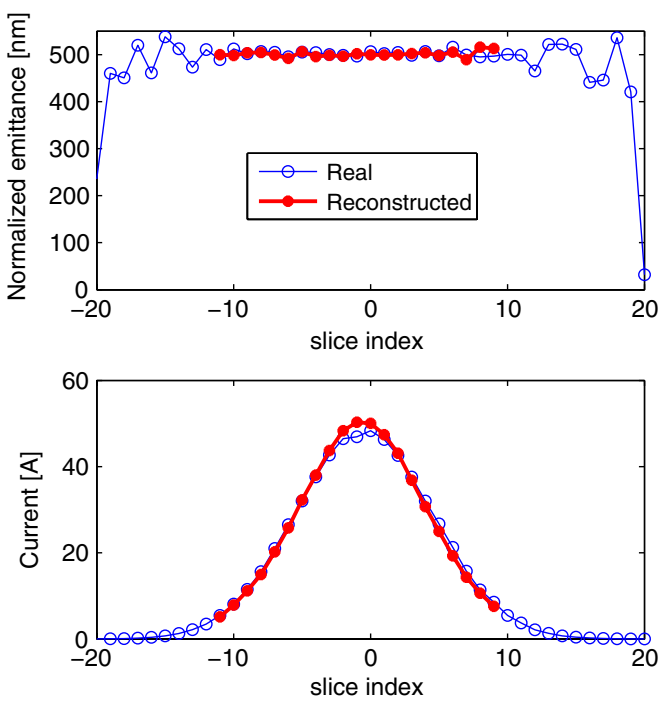

FIG. 6. Simulation of a horizontal slice-emittance measurement. The upper and lower plots show the normalized slice emittance and the current profile, respectively. The blue curves with open points correspond to the real simulated values, the red curves with full points to the reconstructed values.

A Gaussian distribution with $2 \times 10^{5}$ particles is used as an input for the simulations. The beam charge is $200 \mathrm{pC}$, the energy is $130 \mathrm{MeV}$, the bunch length is $0.5 \mathrm{~mm}$, and the normalized emittance is $500 \mathrm{~nm}$. The correlated energy spread is $1 \%(\mathrm{rms})(\delta=0.01)$.

Figure 6 shows simulation results in the case where the measurement is performed in the horizontal plane by generating a vertical orbit bump. We see a very good agreement between the real and reconstructed normalized emittances and longitudinal profiles. Similar agreement was observed for the vertical slice-emittance measurement.

\section{E. Measurement examples}

We have performed the described horizontal and vertical slice-emittance measurements. For the horizontal plane, we have compared the results with a measurement using the TDS.

In all our measurements we divide the beam into 5 slices per rms bunch length. For each image the centroid and size of the total bunch as well as the size for each slice are obtained by means of Gauss fits on the full data set. We subtract the background to remove the influence of unwanted contributions, e.g., dark-current, noise in the camera system, and damaged pixels. We use an average of typically 10 background images.

As in the simulations, the correlated energy spread was set to about $1 \%$. We use the energy spectrometer to find the zero crossing and to set the value of the energy chirp. The dispersion was generated as described before. Figure 7 shows an example of streaked beam in both directions using dispersion. 

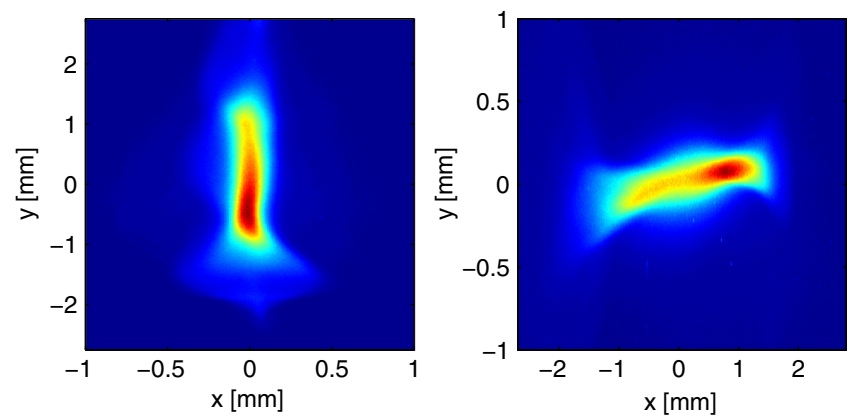

FIG. 7. Example of a streaked beam using dispersion in the vertical direction (left plot) and in the horizontal plane (right plot).

Figure 8 shows the results of the horizontal sliceemittance measurements. We performed four measurements, one with the TDS, two using dispersion and another one with the TDS (in chronological order). The sliceemittance measurement with the TDS is done in a similar way as the dispersion-based measurement: five quadrupoles are used to generate the required phase advance in $x$, to optimize the longitudinal resolution, and to keep the beam sizes at the measurement location under controlmore information can be found in Ref. [25]. The image analysis applied for the TDS measurements is the same as the one applied for the dispersion-based measurements.
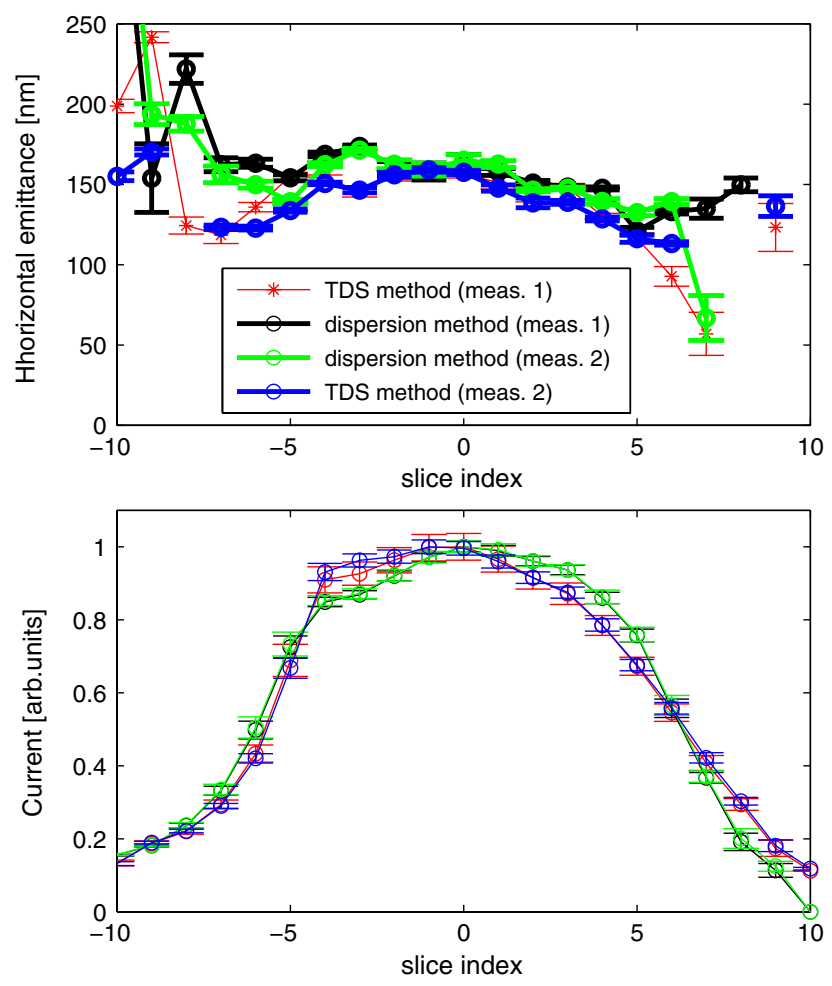

FIG. 8. Horizontal slice-emittance measurement. The upper plot shows the normalized slice emittance as a function of the slice index, the lower plot the relative current profile of the beam.
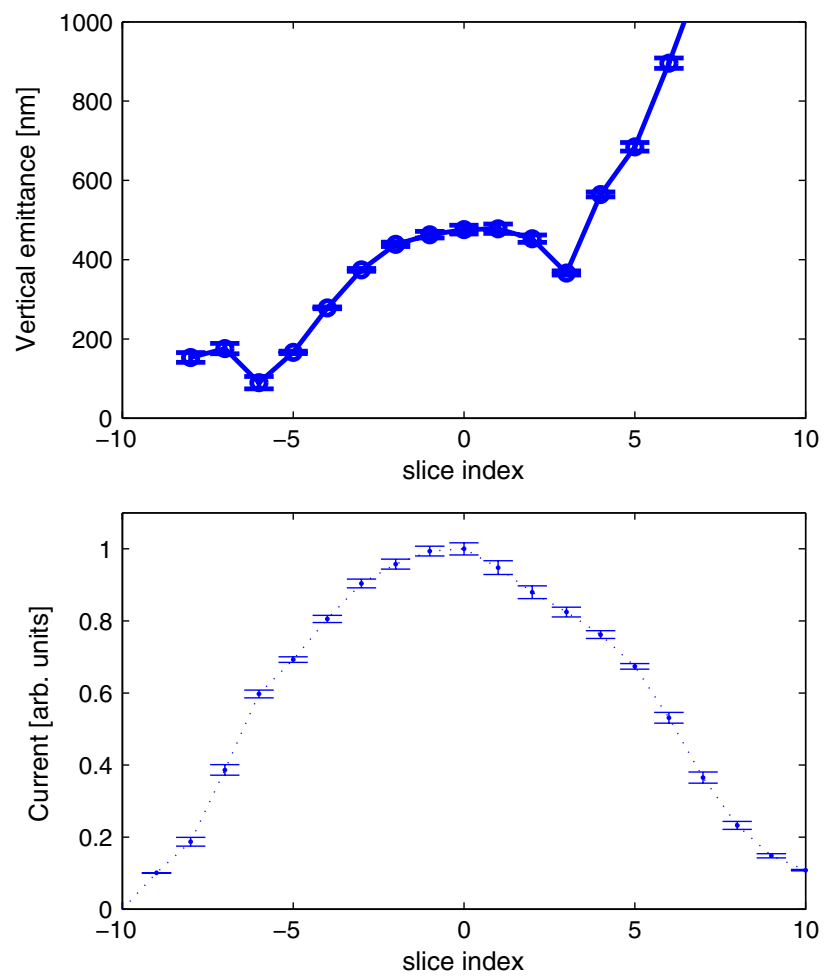

FIG. 9. Vertical slice-emittance measurement. Normalized emittance (upper plot) and current (lower plot) as a function of the slice index.

The error bars shown in the plots correspond to the statistical uncertainties of the measurement. As discussed in Sec. III C for the dispersion-based measurements and in Ref. [25] for the TDS measurements, the systematic errors of the measured emittances are estimated to be well below $5 \%$. The beam charge was about $40 \mathrm{pC}$, the beam energy was $140 \mathrm{MeV}$, and the normalized horizontal projected emittance was about $220 \mathrm{~nm}$. We find good agreement between the dispersion-based and the TDS measurements.

Figure 9 shows the results of the vertical slice-emittance measurements. The energy was $180 \mathrm{MeV}$, the beam charge was $200 \mathrm{pC}$, and the normalized vertical projected emittance was about $700 \mathrm{~nm}$. Since at the SITF the TDS deflects the beam in the vertical direction, a measurement of the slice emittance in $y$ is not possible using this method.

\section{CONCLUSION}

We have presented a method to measure the slice beam parameters in both transverse directions by introducing dispersion to energy-chirped beams. Our generalized approach to generate the dispersion enables measurements in both planes at almost any location in addition to the dedicated dispersive sections. Although the dispersionbased method only provides relative time information, longitudinal diagnostics, or some other technique such as the rf-phasing method may be used to obtain absolute 
time information. We have shown with simulations and measurements that our method can be used to reconstruct the slice emittance and current profile of the beam with high precision. In particular, we have experimentally validated our results through a direct comparison to results obtained with the well-established TDS technique.

Our method replaces the TDS, which is usually expensive to acquire and to operate, by readily available standard accelerator components and has the significant additional benefit of extending the applicability of the measurement to almost any location along the accelerator as well as to both transverse planes. If the facility already has a TDS, our method serves as a cross-check for the TDS technique in the TDS measurement plane and as a complementary measurement in the other transverse plane and at other locations.

\section{ACKNOWLEDGMENTS}

The authors would like to thank Sven Reiche and Thomas Schietinger for helping to improve the content and the language of the manuscript.

[1] R. Ganter et al., PSI Report No. 10-04, 2012.

[2] P. Emma, J. Frisch, and P. Krejcik, SLAC Report No. LCC0047 (LCLS-TN-00-12), 2000.

[3] J. Arthur et al., SLAC Report No. R-593, 2002.

[4] SCSS X-FEL R\&D Group, SCSS X-FEL Conceptual Design Report, edited by T. Shintake and T. Tanaka (RIKEN Harima Institute/SPring-8, Sayo, Japan, 2005).

[5] C. J. Bocchetta et al., Elettra Report No. ST/F-TN-07/12, 2007.

[6] M. Altarelli et al., DESY Report No. 2006-097, 2007.

[7] M. Röhrs, PhD thesis, University of Hamburg, 2008.

[8] C. Behrens, PhD thesis, University of Hamburg, 2012.

[9] D. H. Dowell, P. R. Bolton, J. E. Clendenin, P. Emma, S. M. Gierman, C. G. Limborg, B. F. Murphy, J. F. Schmerge, and W. S. Graves, SLAC Report No. 9540, 2002.

[10] Y. Ivanisenko, PhD thesis, University of Hamburg, 2012.
[11] X. Qiu, K. Batchelor, I. Ben-Zvi, and X-J. Wang, Phys. Rev. Lett. 76, 3723 (1996).

[12] S. Di Mitri, D. Castronovo, I. Cudin, and L. Fröhlich, Phys. Rev. ST Accel. Beams 16, 042801 (2013).

[13] K. Bertsche, P. Emma, and O. Shevchenko, in Proceedings of the 23rd Particle Accelerator Conference, Vancouver, Canada, 2009 (IEEE, Piscataway, NJ, 2009), p. 2346.

[14] P. Emma, C. Behrens, Z. Huang, and F. Zhou, in Proceedings of the 34th International Free-Electron Laser Conference, Nara, Japan, 2012 (JACoW, Geneva, 2013), p. 349.

[15] D. H. Dowell, P. R. Bolton, J.E. Clendenin, S. M. Gierman, C. G. Limborg, B. F. Murphy, J. F. Schmerge, and T. Shaftan, Nucl. Instrum. Methods Phys. Res., Sect. A 507, 331 (2003).

[16] H. Loos, P. R. Bolton, J. E. Clendenin, D. H. Dowell, S. M. Gierman, C. G. Limborg, J. F. Schmerge, T. V. Shaftan, and B. Sheehy, Nucl. Instrum. Methods Phys. Res., Sect. A 528, 189 (2004).

[17] P. Emma, C. Behrens, and H. Loos, in Proceedings of the 34th International Free-Electron Laser Conference, Nara, Japan, 2012 (JACoW, Geneva, 2013), p. 602.

[18] M. Pedrozzi et al., PSI Report No. 10-05, 2010.

[19] K. L. Brown, SLAC Report No. 75, 1982.

[20] J. Rossbach and P. Schmüser, in Proceedings of the 5th CAS general accelerator physics course, Jyväskylä, 1992, edited by S. Turner (CERN, Geneva, 1992), p. 17.

[21] C. Gulliford, A. Bartnik, I. Bazarov, L. Cultrera, J. Dobbins, B. Dunham, F. Gonzalez, S. Karkare, H. Lee, H. Li, Y. Li, X. Liu, J. Maxson, C. Nguyen, K. Smolenski, and Z. Zhao, Phys. Rev. ST Accel. Beams 16, 073401 (2013).

[22] M. W. Guetg, B. Beutner, E. Prat, and S. Reiche, in Proceedings of the 35th International Free-Electron Laser Conference, New York, USA, 2013 (JACoW, Geneva, 2013), p. 267.

[23] M. Borland, APS LS Report No. 287, 2000.

[24] R. Ischebeck (private communication).

[25] E. Prat, M. Aiba, S. Bettoni, B. Beutner, M. Guetg, R. Ischebeck, S. Reiche, and T. Schietinger, in Proceedings of the 35th International Free-Electron Laser Conference, New York, USA, 2013 (JACoW, Geneva, 2013), p. 200. 